\title{
Air pollution monitoring
}

\section{By}

\author{
sensors embedded on mobile phone \\ Nasimkhozouie ${ }^{1}$, FaranakFotouhi-Ghazvini? \\ 1,2 Department of Computer Engineering and IT \\ Qom University \\ Alghadir Boulevard \\ The Old Isfahan Road \\ Qom, Iran Postal Code: 3716146611 \\ N_khozooyi2003@yahoo.com \\ faranak_fotouhi@hotmail.com
}

\section{ABSTRACT:}

Mobile technology has been available for at least a decade and is increasingly being used in developing countries as a way of contacting and connecting citizens and helping them to organize for a better life. ${ }^{[3]}$

Mobile phones are not just for phone calls, but they can also be used to collect data in several different formats and send them to a central server. There the data can be aggregated and analyzed, with tables and visualizations automatically generated. What is new is the sheer number of observation points that are potentially available by using mobile phones. With over 4 billion phones in use worldwide, the mobile phone network is emerging as a form of "global brain" with sensors everywhere. In addition, there are companies such as Fourier Systems that provide purpose-built mobile devices that are specificallydesignedforscienceexperimentsinschoolsandfordatalogginginanyscienceproject. ${ }^{[3]}$

Keywords: Sensor networks; Mobile phone; Air Quality; Air pollution monitoring.

\section{Council for Innovative Research}

Peer Review Research Publishing System

Journal: International Journal Of Computers \& Technology

Vol. 11, No.5

editor@cirworld.com

www.cirworld.com, member.cirworld.com 


\section{Introduction:}

Says Eric Paulos, a researcher at Intel, the world's largest chipmaker. "In a few years, everybody will look at his phone and see what the pollen count is." $[3]$

may be The idea that phones should have sensors is far from outlandish, but Phones already primitive versions, including the sensor that picks up the cellular signal, light sensors that dim the keyboard, and acceleration sensors that notice when the user lifts the phone to his ear. Carbon monoxide, ozone, pollen, sun intensity and temperature are among the things that MrPaulos considers particularly easy to measure by tweaking mobile phones in ways that consumers would not even notice. Any such data would need to be collected in a discreet way to assure the privacy of consumers. But eventually, thinks MrPaulos, this new twist to the everyday mobility of ordinary people could lead to "grassroots citizen science."

\section{Wireless sensor network:}

A wireless sensor network (WSN) consists of spatially distributed autonomoussensors to monitor physical or environmental conditions, such as temperature, sound, pressure, etc. and to cooperatively pass their data through the network to a main location. ${ }^{[6]}$

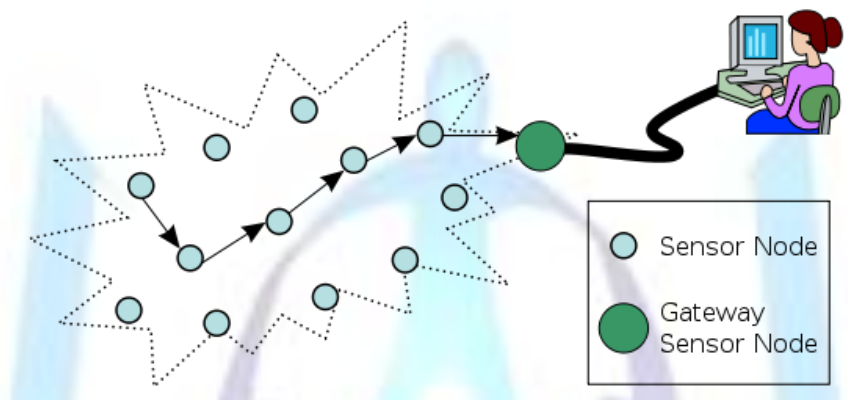

Fig1.Typical multi-hop wireless sensor network architecture

The WSN is built of "nodes" - from a few to several hundreds or even thousands, where each node is connected to one (or sometimes several) sensors. Each such sensor network node has typically several parts:1). a radiotransceiver with an internal antenna or connection to an external antenna, 2).a microcontroller, an electronic circuit for interfacing with the sensors and 3).an energy source, usually a battery or an embedded form of energy harvesting. all parts can embedded on mobile phone, this sensors can feed of mobile battery , and used mobile memory and communication bandwidth .

The topology of the WSNs can vary from a simple star network to an advanced multi-hopwireless mesh network. The propagation technique between the hops of the network can be routing or flooding. [7]

\section{Air quality indicators:}

As a consequence of the frightening increase in air pollution, especially in large urban areas, many international organizations and governments have adopted a series of regulations to keep a low the level of pollutant concentration. Since people incessantly breathe in the atmosphere, the presence of pollutants is very dangerous for human health.

This section analyses and compares three existing Indicators of the air quality: the American AQI, the French ATMO, and the Italian IQA.

For several years, a thorough scientific research has been carried out inorder to find out how much the pollutants diffused in the air affect people.

This activity is carried out monitoring chemically and physically the environment and also using biological indicators to evaluate the impact the pollutants have on the ecosystem and on people(Rapport et al. 2003). For instance, several studies established that nitrogen dioxide (NO2) and ozone $(\mathrm{O} 3)$ increase the risk of death in patients with severe asthma (Sunyeret al.2002). Ozone polluting the air generates an increase in lung cancer (Yang et al. 2005). Traffic-related air pollution increase mortality (Hoek et al. 2002). Ozone and carbon monoxide (CO) are linked to cardiac birth deficiencies(Ritz et al. 2002), etc.

For this purpose, several countries have introduced evaluation methods that rapidly and efficiently indicate the air quality condition for the population. This section analyses and compares three existing Indicators of the air-quality: the American $A Q$, the French ATMO, and the Italian IQA.

\section{The American Air Quality Index (AQI):}

The AQI is used for the Metropolitan Statistical Areas (MSAs) of US, with a population of more than 350,000 - according to the Clean Air Act safetylimits of five air pollutants: Ozone (O3), Particulate Matter (PM), CarbonMonoxide (CO), Sulphur Dioxide (SO2), Nitrogen Dioxide (NO2) USE.P.A., 1999).

For each area, the daily $A Q I$ value is given by the worst registered condition among the five pollutants: 


$$
\mathrm{AQI}=\max \left\{I_{\mathrm{O}_{3}}, I_{\mathrm{PM}_{10}}, I_{\mathrm{CO}}, I_{\mathrm{SO}_{2}}, I_{\mathrm{NO}_{2}}\right\}
$$

Note: the AQI does not fulfil the property of monotony and the AQI does not fulfil any sub-index compensation.

Each AQI value is linked with a color and with an air quality descriptor. The AQI scale is split into six reference categories by E.P.A. - the same reported in Table.

Table1. :American AQI Categories, Descriptors, and Colours (US E.P.A. 1999)

\begin{tabular}{lll}
\hline AQI Reference Values & Descriptor & Colour \\
\hline $0-50$ & Good & Green \\
$51-100$ & Moderate & Yellow \\
$101-150$ & Unhealthy for sensitive groups & Orange \\
$151-200$ & Unhealthy & Red \\
$201-300$ & Very unhealthy & Purple \\
$>301$ & Hazardous & Maroon \\
\hline
\end{tabular}

The E.P.A. qualitative description related to the AQI categories are asfollows:

- Good": the AQI value is within the 0-50 range. The air quality is satisfactory, with very little risk to the population.

- "Moderate": AQI included between 51 and 100. The air quality is admissible,

however a few people could be healthy damaged because of the presence of pollutant.

For instance, ozone sensitive people may experience respiratory symptoms.

- "Unhealthy for sensitive groups": children and adults with respiratory disease are at risk when doing outdoor activities, due to the ozone exposure, whereas people with cardiovascular disease are most at risk due to the carbon monoxide exposure. When the AQI value is included between 101 and 150, these sensitive individuals could increase their symptoms of disease to the point of health compromising. However, much of the population is not at risk.

- Within the 151-200 range the AQI is considered to be "unhealthy". This situation causes possible disease for the general population. Sensitive individuals could seriously suffer.

- "Very unhealthy" AQI values - between 201 and 300 - represent an alarm. The whole population could be health damaged seriously.

- "Hazardous" - over 300 - AQI values trigger an immediate alarm. The whole population is at serious risk of suffering from diseases.

\section{The ATMO index :}

The ATMO index was developed by the French Environment Ministry. It is based on the concentration of four air pollutants: Ozone (O3), Particulate Matter (PM), Sulphur Dioxide (SO2), and Nitrogen Dioxide (NO2) (Ministèredel'écologie et du développement durable 2004;Bouyssouet al.2000).Each of the pollutants is related to a subindex. ${ }^{[1]}$

Table 2.Ten reference categories of the four sub-indexes which make up the

ATMO indicator. Each level is set out between a minimum and a maximum

BreakpointValue (Ministère de l'écologieet du

développement durable 2004)

\begin{tabular}{|c|c|c|c|c|c|c|c|c|c|c|}
\hline \multirow[t]{2}{*}{ Lev. } & \multicolumn{2}{|c|}{$\begin{array}{l}\mathrm{PM}_{10} \\
{\left[\mu \mathrm{g} / \mathrm{m}^{3}\right]}\end{array}$} & \multicolumn{2}{|c|}{\begin{tabular}{|l|}
$\mathrm{NO} 2$ \\
{$\left[\mu \mathrm{g} / \mathrm{m}^{3}\right]$}
\end{tabular}} & \multicolumn{2}{|c|}{$\begin{array}{l}\mathrm{O}_{3} \\
{\left[\mu \mathrm{g} / \mathrm{m}^{3}\right]}\end{array}$} & \multicolumn{2}{|c|}{$\begin{array}{l}\mathrm{SO}_{2} \\
{\left[\mu \mathrm{g} / \mathrm{m}^{3}\right]}\end{array}$} & \multirow[t]{2}{*}{ Descriptor } & \multirow[t]{2}{*}{ Colour } \\
\hline & Min & Max & Min & Max & Min & Max & Min & Max & & \\
\hline 1 & 0 & 9 & 0 & 29 & 0 & 29 & 0 & 39 & Very Good & Green \\
\hline 2 & 10 & 19 & 30 & 54 & 30 & 54 & 40 & 79 & Very Good & Green \\
\hline 3 & 20 & 29 & 55 & 84 & 55 & 79 & 80 & 119 & Good & Green \\
\hline 4 & 30 & 39 & 85 & 109 & 80 & 104 & 120 & 159 & Good & Green \\
\hline 5 & 40 & 49 & 110 & 134 & 105 & 129 & 160 & 199 & Medium & Orange \\
\hline 6 & 50 & 64 & 135 & 164 & 130 & 149 & 200 & 249 & Poor & Orange \\
\hline 7 & 65 & 79 & 165 & 199 & 150 & 179 & 250 & 299 & Poor & Orange \\
\hline 8 & 80 & 99 & 200 & 274 & 180 & 209 & 300 & 399 & $\mathrm{Bad}$ & Red \\
\hline 9 & 100 & 124 & 275 & 399 & 210 & 239 & 400 & 499 & Bad & Red \\
\hline 10 & $\geq 12$ & & $\geq 40$ & & $\geq 24$ & & $\geq 50$ & & Very Bad & Red \\
\hline
\end{tabular}


The ATMO value is the maximum of the four sub-indexes:

$$
\mathrm{ATMO}=\max \left\{I_{\mathrm{NO}_{2}}, I_{\mathrm{SO}_{2}}, I_{\mathrm{O}_{3}}, I_{\mathrm{PM}_{10}},\right\}
$$

- Note: the ATMO does not fulfill the property of monotony. ${ }^{[1]}$

\section{The IQA index (Italian Index for Air Quality):}

In some northern Italian regions (Piedmont, Lombardy, etc.) different systems are currently being tested to monitor the air quality and provide information to the public.

The index is inspired by the United States Environmental Protection Agency, AQI but with some clear differences.

According to the safety regulation limit, the IQA aggregates the most critical air pollutants at each time of the year on the basis of their effects on human health: Ozone $\left(\mathrm{O}_{3}\right)$ and Particulate Matter (PM10) in summertime,PM10and Nitrogen Dioxide (NO2) in wintertime.

The IQA value is the arithmetic mean of the two maximum sub-indexes' values:

$$
\mathrm{IQA}=\frac{I_{1}+I_{2}}{2}
$$

where I 1and Izare the two sub-indexes with the higher value (selected among the three criticalpollutants PM10, O3, $\mathrm{NO}_{2}$ ). The IQA is calculated on a daily basis using the values of the concentration of pollutants from the previous 24 hours.

\begin{tabular}{llll}
\multicolumn{2}{|c}{ Table3. IQA Categories, Descriptors and Colours (Piedmont Regional law } \\
\multicolumn{4}{|c|}{$\mathbf{4 3 0 0 0}$ 2000) } \\
\hline IQA reference values & IQA final rank & Descriptor & Colour \\
\hline $0-50$ & 1 & Excellent & Blue \\
$51-75$ & 2 & Good & Light blue \\
$76-100$ & 3 & Fair & Green \\
$101-125$ & 4 & Mediocre & Yellow \\
$126-150$ & 5 & Not very healthy & Orange \\
$151-175$ & 6 & Unhealthy & Red \\
$>175$ & 7 & Very unhealthy & Purple \\
\hline
\end{tabular}

Different descriptions of the air quality, different colors, and some useful advice for the population are associated with each of the seven IQA levels:

- "Excellent" - blue, with a numeric IQA value between 0 and 50 . Thequality of the air is considered excellent.

- "Good" - light blue, with a numeric IQA value between 51 and 75 . The air quality is considered very satisfactory with no risk for the population.

- "Fair" - green, with a numeric IQA value between 76 and 100. The air quality is satisfactory and there is no risk for the population.

- "Mediocre" - yellow, with a numeric IQA value between 101 and 125. The population is not at risk. People with asthma, chronic bronchitis or heart problems might show symptoms of slight breathing problems, but only during intense physical activity; it is advised that people with these ailments category of people limit their physica exercise outdoors, especially during the summertime.

- "Not very healthy" - orange, with a numeric IQA value between 126and 150. People with heart problems, the elderly and children may be atrisk. It is advised that these categories of people limit their physical activityand prolongedperiodsof timeoutdoors, especially during thepeakdaytime hours insummertime.

- "Unhealthy" - red, with a numeric IQA value between 151 and 175.Many people could have slightly negative health problems, albeit reversible;it is advisedto limitextended periodsof timeoutdoors,especiallyinthe peakdaytime hours duringthesummertime. People in thesensitivegroups could, however, may havemoreserious symptoms;in thesecasesit is highly recommended exposeoneself as little as possible to theopenair.

- "Very unhealthy" - purple, with a numeric IQA value above 175. Theremay be slightly negative effects on the health of all people in the area.The elderly and people with respiratory problems (breathing difficulties)should avoid going outside. Other people (especially children) shouldavoid doing physical activity and limit their time outdoors, especiallyduring the peak daytime hours in summertime. 


\section{Air pollution monitoring by sensorsembedded on mobile phone :}

Wireless sensor networks have been deployed in several cities (Stockholm, London and Brisbane) to monitor the concentration of dangerous gases for citizens. These can take advantage of the ad hoc wireless links rather than wired installations, which also make them more mobile for testing readings in different areas. There are various architectures that can be used for such applications as well as different kinds of data analysis and data mining that can be conducted. [2]

Although we should be rightly concerned about invasion of individual privacy through surveillance by mobile devices, there is also tremendous potential in using individual mobile devices as sensing nodes in a larger network. ${ }^{[3]}$ For example, the Economist (2008) reports that researchers at Purdue University revealed that they are constructing a network of mobile phones to detect and track radiation. A nuclear leak or a "dirty bomb" would set off the sensors in a large number of phones that would then identify their location through GPS. ${ }^{[3]}$

All embedded sensors of a mobile phone can be subdivided into two categories. The firstone includes environment sensors and the other contains position and orientation sensors. Environment sensors are used to measure different properties of the mobile phone environment. ${ }^{[8]}$ such as the amount of nitrogen dioxide (NO2) and ozone (O3), Particulate Matter (PM10), Carbon Monoxide (CO)and etcof air.

Microphone and camera are examples of the first sensor group. Such sensors as accelerometer,digital compass, gyroscope and GPS form position and orientation sensors group. They areused to determine orientation of the mobile phone in space and also device location.Prevalence of these sensors make them attractive for various sensing applications,such as automatic phone context detection. [8]

The other environment sensors is a light sensor and a proximity sensor. The light sensor measures intensity of ambient light and can beused to adjust brightness of the screen. The proximity sensor detects presence of nearby objects. When the user holds the phone close to his or her ear to answer the call, the screen is blocked to prevent accidental touch. [9]

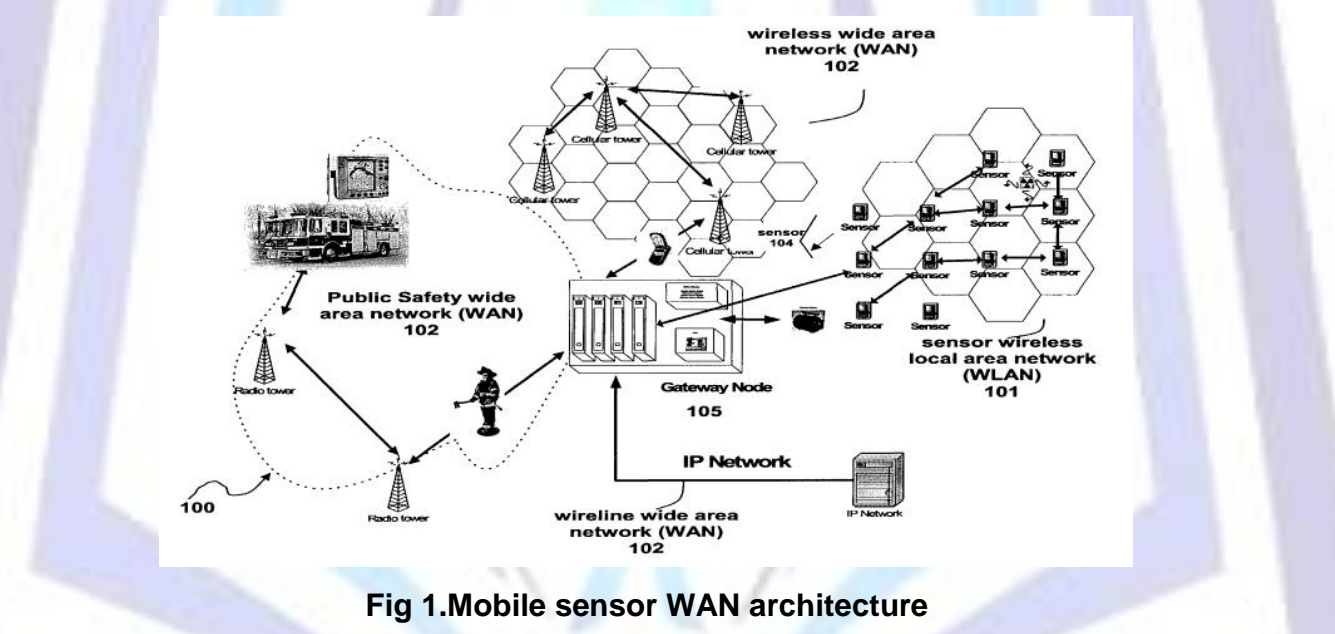

mobile phone sensing architecture that comprises the following building blocks. ${ }^{[10]}$

SENSE

Individual mobile phones collect raw sensor data from sensors embedded in the phone.

\section{LEARN}

Information is extracted from the sensor data by applying machine learning and data mining techniques.

These operations occur either directly on the phone, in the mobile cloud, or with some partitioning between the phone and cloud. Where these components run could be governed by various architectural considerations, such as privacy, providing user real-time feedback, reducing communication cost between the phone and cloud, available computing resources, and

sensor fusion requirements. We therefore consider where these components run to be an open issue that requires research.

\section{INFORM, SHARE, AND PERSUASION}

We bundle a number of important architectural components together because of commonality or coupling of the components. For example, a personal sensing application will only inform the user ,whereas a group or community sensing application may share an aggregate version of information with the broader population and obfuscate the identity of the users. Other considerations are how to best visualize sensor data for consumption of individuals,groups, and communities. Privacy is a very important consideration as well.. ${ }^{[10]}$ 


\section{Assessment Air pollution by mobile phone:}

Online collaborative sensor data management platforms are on-line database services that allow sensor(mobile) owners to register and connect their devices to feed data into an online database for storage and also allow developers to connect to the database and build their own applications based on that data. Examples include Xively and the Wikisensing platform. The architecture of this platform is described the key components of such systems to include APIs and interfaces for online collaborators, a middleware containing the business logic needed for the sensor data management and processing and a storage model suitable for the efficient storage and retrieval of large volumes of data. [4]

\section{Personal assessment Air pollution:}

Such platforms simplify online collaboration between users over diverse data sets ranging from energy and environment data that collected from sensors. Other services include allowing developers to embed real-time graphs \& widgets in websites; analysis and process air pollution data according Air quality indicators and send real-time alerts to owner mobile by sms or rich media file depend on mobile phone.

\section{Public assessment Air pollution:}

Set off the sensors in a large number of phones can send data of air and GPS data to a common Web database. There the data can be displayed and analyzed, along with previously collected data, and then plotted on GoogleMaps. Alternatively air data and GPS data analyzed, and Air Quality of every zone according tables[1,2,3] be determined to inform people who come to the area by mobile or any other means of communication , such as radio,...

\section{Challenges:}

Thereis little or no consensus on the sensing architecturefor the phone and the cloud. For example,newtools new tools and phone software will be needed tofacilitatequick development and deployment ofrobustcontext

classifiers for the leading phonesonthe market. Common methods for collectingandsharing data need to be developed. Mobilephonescannot be overloaded with continuoussensingcommitments that undermine the performanceof the phone. ${ }^{[10]}$ But The most important problems are security, privacy, user trust, and legal compliance with existing data protection laws. ${ }^{[5]}$ more over ,Encourage people to use this service, register and connect their devices to feed data into an online database for storage and also allow developers to connect to the database and build their own applications based on that data.

\section{REFRENCES:}

1. FiorenzoFranceschini, Maurizio Galetto,DomenicoMaisano,Management by Measurement, Designing Key Indicators and Performance Measurement Systems,2007

2. Ma, Y.; Richards, M.; Ghanem, M.; Guo, Y.; Hassard, J. (2008). "Air Pollution Monitoring and Mining Based on Sensor Grid in London". Sensors 8 (6): 3601. doi:10.3390/s8063601

3. GARY WOODILL, ED.D,THE MOBILE LEARNING EDGE,2010

4. Silva, D.; Ghanem, M.; Guo, Y. (2012). "WikiSensing: An Online Collaborative Approach for Sensor Data Management". Sensors 12 (12): 13295. doi:10.3390/s121013295

5. Alan S. Weber, "Cloud Computing in Education", Ubiquitous and Mobile Learning in the Digital Age,2013

6. ^Dargie, W. and Poellabauer, C., "Fundamentals of wireless sensor networks: theory and practice", John Wiley and Sons, 2010 ISBN 978-0-470-99765-9, pp. 168-183, 191-192

7. Jump up ^Sohraby, K., Minoli, D., Znati, T. "Wireless sensor networks: technology, protocols, and applications, John Wiley and Sons", 2007 ISBN 978-0-471-74300-2, pp. 203-209

8. EvgenyStankevich, IlyaParamonov, Ivan Timofeev,"Mobile Phone Sensors in Health Applications",PROCEEDING OF THE 12TH CONFERENCE OF FRUCT ASSOCIATION,ISSN 2305-7254,136141

9. K. Hinckley, J. Pierce, M. Sinclair, and E. Horvitz, Sensing Techniques for Mobile Interaction, in Proceedings ofACMUIST, 2000, pp. 91100.

10. Nicholas D. Lane, EmilianoMiluzzo, Hong Lu, Daniel Peebles, TanzeemChoudhury,

and Andrew T. Campbell, Dartmouth College, A Survey of Mobile Phone Sensing ,IEEECommunications Magazine - September 2010,p140-150 